\title{
Commissioning and Validation of the ATLAS Level-1 Topological Trigger in Run 2
}

\author{
Daniel Zheng \\ Department of Electrical and Computer Engineering, University of Pittsburgh, \\ Pittsburgh, PA, 15213, USA \\ E-mail: daniel.zheng@pitt.edu \\ Andrew Aukerman \\ Department of Physics and Astronomy, University of Pittsburgh, \\ Pittsburgh, PA, 15213, USA \\ E-mail: ataukerman@pitt.edu \\ Tae Min Hong \\ Department of Physics and Astronomy, University of Pittsburgh, \\ Pittsburgh, PA, 15213, USA \\ E-mail: tmhong@pitt.edu
}

On behalf of the ATLAS Collaboration

The ATLAS experiment has introduced and recently commissioned a completely new hardware sub-system of its first-level trigger: the topological processor (L1Topo). L1Topo consist of two AdvancedTCA blades mounting state-of-the-art FPGA processors, providing high input bandwidth (up to $4 \mathrm{~Gb} / \mathrm{s}$ ) and low latency data processing (200 ns). L1Topo is able to select collision events by applying kinematic and topological requirements on candidate objects (energy clusters, jets, and muons) measured by calorimeters and muon sub-detectors. Results from data recorded using the L1Topo trigger will be presented. These results demonstrate a significantly improved background event rejection, thus allowing for rate reduction with minimal efficiency loss. This improvement has been shown for several physics processes leading to low- $p_{T}$ leptons, including $H \rightarrow \tau \tau$ and $J / \psi \rightarrow \mu \mu$. In addition to describing the L1Topo trigger system, we will discuss the use of an accurate L1Topo simulation as a powerful tool to validate and optimize the performance of this new system. To reach the required accuracy, the simulation must mimic the approximations applied in firmware to execute the kinematic calculations.

\section{Introduction}

The ATLAS Experiment at the European Organization for Nuclear Research (CERN) is one of two general purpose experiments at the Large Hadron Collider (LHC) in Meyrin, Switzerland. The LHC collides proton bunches with a frequency of $40 \mathrm{MHz}$. 1 The ATLAS Trigger and Data Acquisition (TDAQ) system discards collision events that are not of physics interest. ${ }^{2}$ As the LHC operates at increasing instantaneous luminosity, the need for further rate reduction arises. 


\subsection{TDAQ}

The TDAQ system is comprised of two levels, the first being a hardware based, low-granularity Level-1 (L1) system with a fixed latency of $2.5 \mu \mathrm{s} . \underline{3}$ This system constructs Regions-of-Interest seeding the software based algorithms used in the subsequent High-Level Trigger (HLT) system, reconstructing the event with full detector read-out granularity. The L1 system is further divided into three subcomponents: the Calorimeter Trigger (L1Calo), the Muon Trigger (L1Muon) and the Central Trigger Processor (CTP). The L1 system reduces the $40 \mathrm{MHz}$ collision rate to $100 \mathrm{kHz}$, which is further reduced by the HLT trigger to $3-4 \mathrm{kHz} \cdot \underline{3}$ The Level-1 Topological Trigger (L1Topo) system is a new component of the ATLAS TDAQ system that makes it possible to impose topological constraints to L1 triggers. ${ }^{4}$ The goal of this system is to improve L1 event selection by imposing kinematic requirements based on the event topology of certain processes. Its electronic boards compute angular and kinematic quantities between various L1 Trigger Objects (TOBs) ${ }^{4}$ Its role in the L1 trigger is shown in the flowchart included in Fig. 1 . The system is designed to receive and process up to $6 \mathrm{~Tb} / \mathrm{s}$ of real time data and was commissioned and validated in the $2017 \mathrm{LHC}$ run at $\sqrt{s}=7 \mathrm{TeV}$. 4

\section{Motivation}

As higher luminosities are reached at the LHC, the production rate for physics signatures increases. Traditionally, this is dealt with using prescales, which save only 1 of every $n$ events that pass the trigger, or by tightening selection criteria, e.g., by increasing the transverse momentum threshold required. ${ }^{5}$ Neither of these approaches is optimal, however, as they both cause data loss that affects particular studies involving high- $p_{T}$ objects. There are a variety of physics analyses that benefit from the introduction of topological cuts. For example, L1Topo provides rate reduction for the $B^{0} \rightarrow J / \psi \phi$ analysis, which was reaching unsustainable rates for low $p_{T}$ muon triggers. $\frac{6}{6}$

\section{L1Topo Algorithm Execution}

\subsection{L1Muon}

In Run 1, the Muon CTP Interface (MUCTPI) extracted only muon multiplicity and momentum quantities for the Central Trigger Processor (CTP) to use in the L1Muon decision. ${ }^{2}$ With the introduction of L1Topo, additional kinematic and angular cuts can be performed. 4 Thus, for Run 2, the MUCTPI was upgraded to also extract coarse-grained muon candidates to send to the L1Topo module. ${ }^{7}$ The MUCTPI system includes 16 Muon Octant (MIOCT) modules, one for each octant in each half of the detector. Up to 32 muon candidates are sent to L1Topo. A feasibility study in which a high resolution phase scan was performed on data received from the overclocked MIOCT modules showed that there exists an optimal sampling point for DDR data transmission, with a bit error rate lower than 10-15 at 95\% confidence 


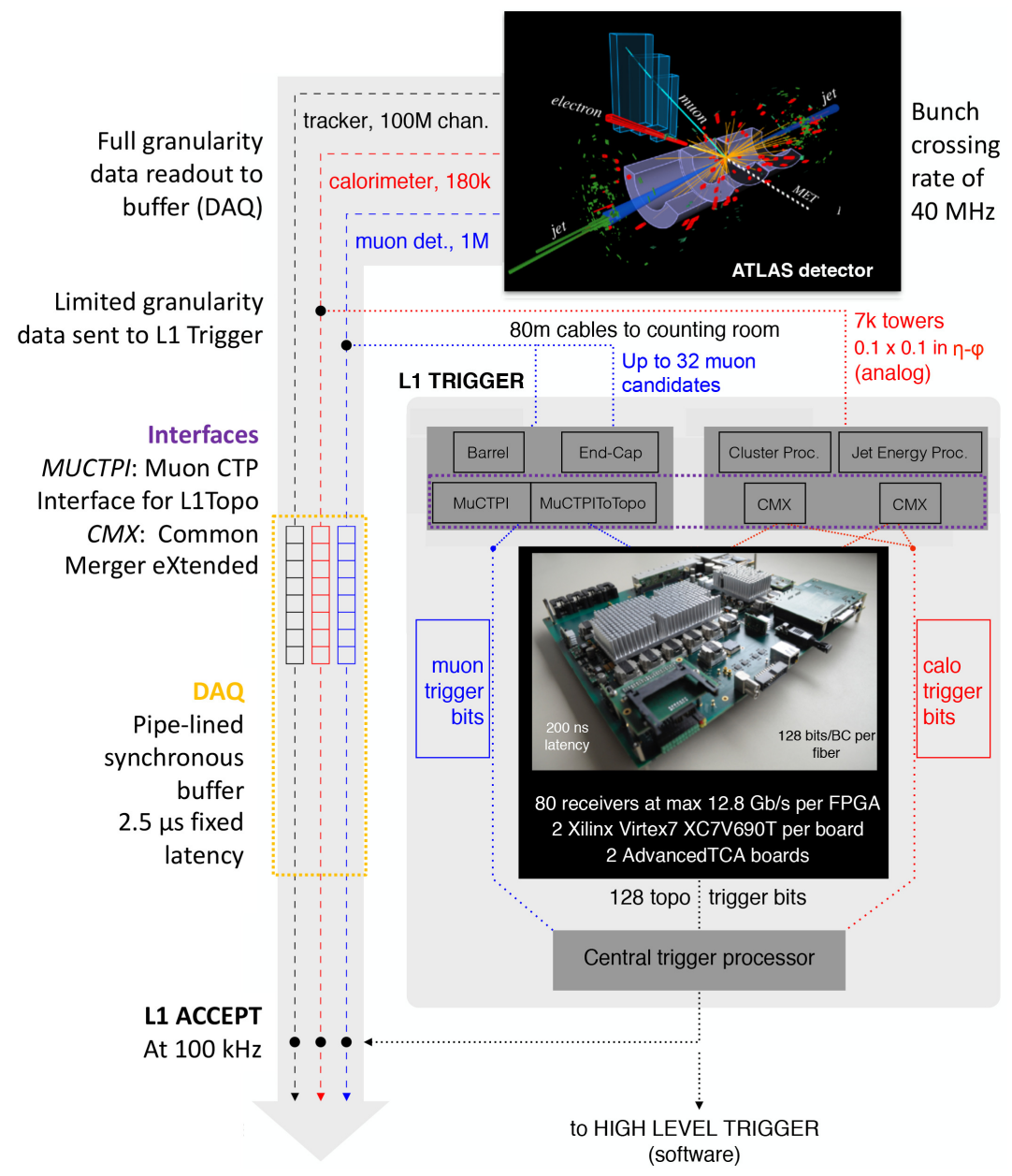

Fig. 1. Data flow through the L1 Trigger System with emphasis on L1Topo's role.

level. ${ }^{7}$ This demonstrates that the MUCTPI trigger outputs can reliably transmit data to L1Topo. The total aggregated data rate of the MUCTPI system is 10.24 $\mathrm{Gb} / \mathrm{s} \cdot{ }^{7}$ Commissioning tests of this system specifically include use of a high-level software simulation to generate input vectors and calculation of expected results. ${ }^{7}$ No errors appeared in a sample of snapshot memory from 1010 bunches. The parallel output from the MUCTPI must also be encoded into optical output for the L1Topo modules. This is implemented in a dedicated MUCTPIToTopo board, which includes a Xilinx VC707 development kit and two FMC cards. Integration tests showed zero errors out of $2.6 * 10^{6}$ test bunches. ${ }^{7}$ 


\subsection{L1Calo}

The L1Calo Common Merger Modules (CMMs), which interface with the CTP, had to be replaced to run at higher bandwidth and to function with the proposed L1Topo module. The CMM modules, in the same vein as the MIOCT modules, collect information from the calorimeter specific to their crate numbers. ${ }^{8}$ The upgrade of these crate modules to Common Merger eXtended (CMX) models allows transmission of $120 \mathrm{e} / \gamma$ candidates, $120 \tau$ candidates, 64 jet candidates, and MET information to L1Topo.

\subsection{L1Topo}

The L1Topo system is a crate equipped with two AdvancedTCA L1Topo modules. In each module, TOB data is received from the L1Calo and L1Muon systems through optical ribbon fibers in the backplane and front panel. These optical signals are then converted to electrical ones and transmitted to two Virtex-7 FPGAs. Algorithms are executed within $75 \mathrm{~ns}$, corresponding to three LHC bunch crossings. The first two ticks are used for data reduction, consisting of sorting TOBs by $E_{t}$ value or a selection algorithm providing an $E_{t}$ cut. Decision algorithms are subsequently applied to the reduced input lists and output bits are sent to the CTP for use in the L1 trigger decision. The output consists of a decision and an overflow bit. The overflow sets the decision to true so the HLT can examine the event with more granular information and complex algorithms. The module can handle a maximum of 128 trigger algorithms (32 per FPGA).

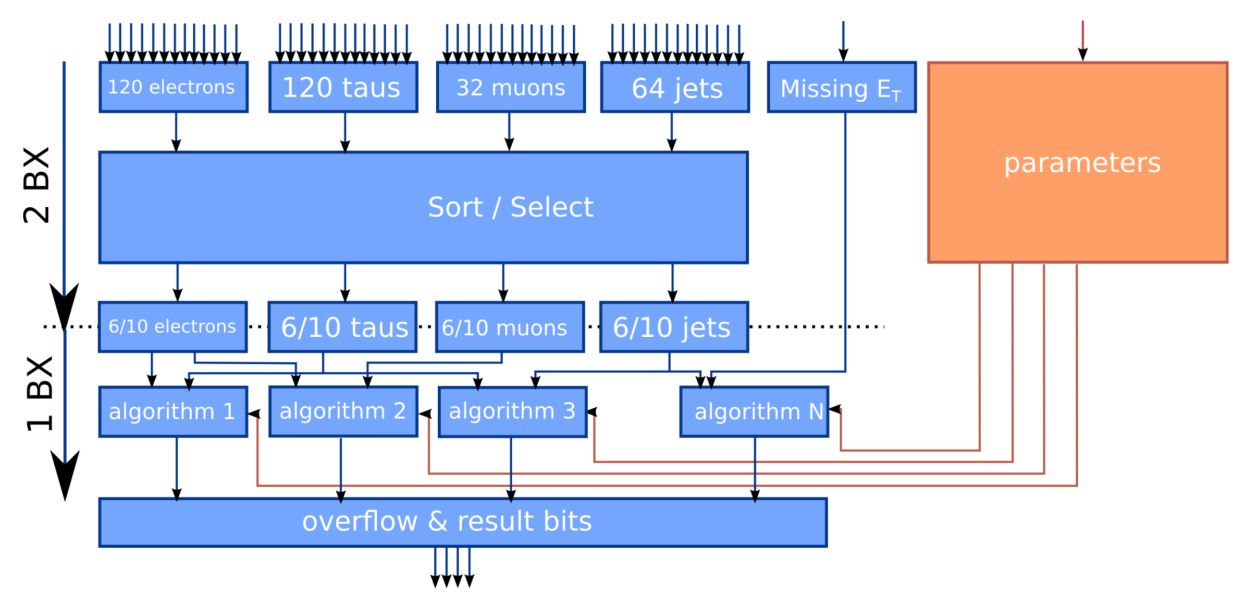

Fig. 2. Diagram of the L1Topo algorithm execution. 9 


\section{Commissioning}

The goal L1Topo is to improve the purity of L1 trigger selection by imposing kinematic and angular constraints. This results in a rate reduction when the rate for a L1 trigger including a topological requirement is compared with the one for the same multiplicity and energy thresholds, but without the topological requirement. An example of this rate reduction is for the case of a trigger targeting low-mass dimuon resonances. In this case the topological requirements are that the two muons are collinear, $0.2<\Delta R(\mu, \mu)<1.5$, and the muons have an invariant mass consistent with the resonance, $2 \mathrm{GeV}<m(\mu, \mu)<9 \mathrm{GeV}$ (Fig. 3). This rate reduction is achieved without significant loss in the selection efficiency with respect to the L1 trigger requiring only two muons with $p_{T}>6 \mathrm{GeV}$.4

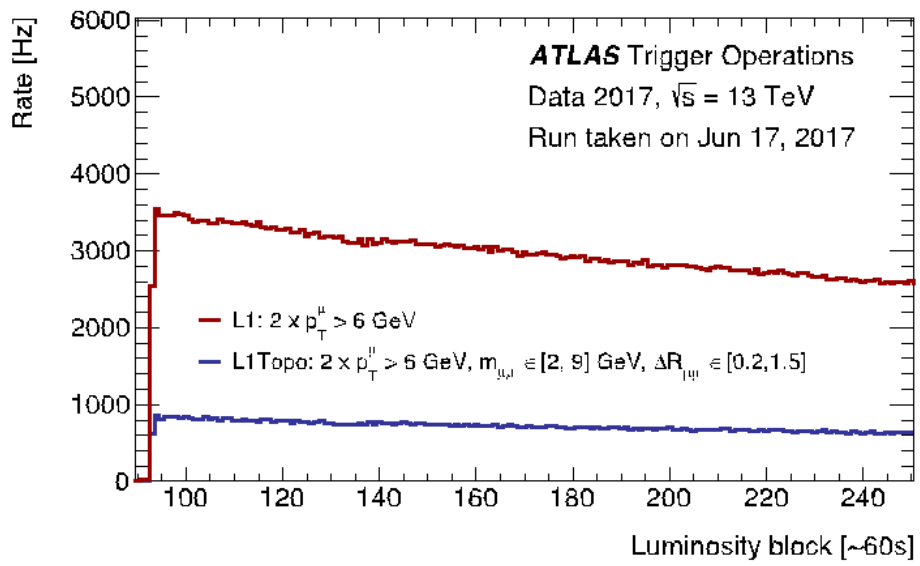

Fig. 3. The rate vs. time of two triggers selecting two muons with transverse momentum larger than $6 \mathrm{GeV}$. The L1Topo trigger rate (blue) includes the additional topological requirement that the two L1 muons form an invariant mass $2 \mathrm{GeV}<M_{\mu \mu}<9 \mathrm{GeV}$ and have angular separation $0.2<\Delta R<1.5$, showing a significant rate reduction. 5

\subsection{Xmon Online Rate Monitoring}

The Xmon rate monitoring system is a tool available to trigger shifters and experts. Xmon provides real-time, luminosity scaled rate predictions using pileup linear regressions unique to each trigger. Each trigger can be written in terms of cross-section by considering the current rate of the trigger and the instantaneous luminosity and adjusting for number of bunches and prescale. The detected pileup is used to perform a linear regression to estimate this cross section. The estimated parameters are then used in real time and multiplied by luminosity to predict trigger rates. For L1Topo, an additional functionality was added to the Xmon tool to monitor the ratios between trigger rates. Specifically, the ratio of L1Topo items 
with their L1 equivalent without topological requirements. This in turn allows for the fast detection of potential issues arising with the MUCTPI, CMX modules, CTP link, and the L1Topo modules themselves. Issues involving L1Topo trigger rates would immediately be visible if they involved the new L1Topo components. An example showing the ratio between the L1Topo rate and L1 rate for muons is shown in Fig. 4

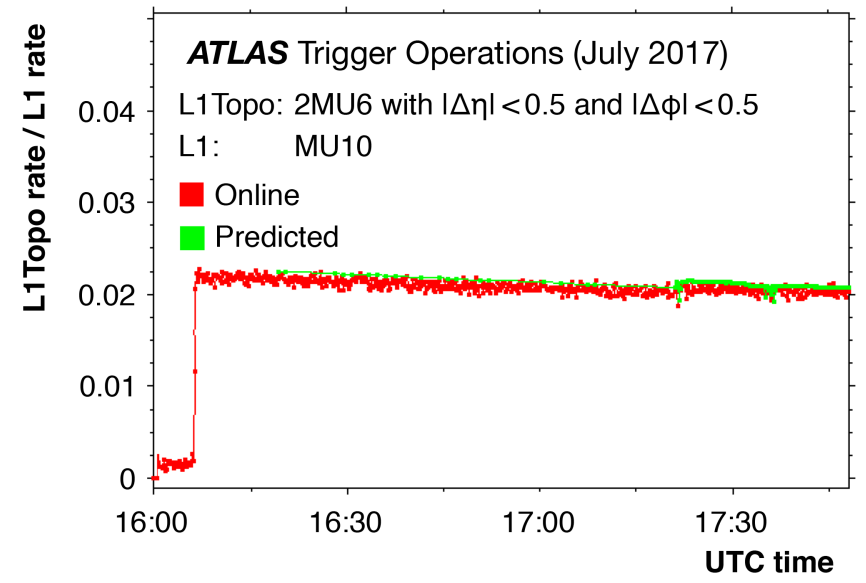

Fig. 4. L1Topo rate ratio monitoring. The rate of the L1Topo trigger requiring two muons with $p_{T}>6 \mathrm{GeV}$ and $|\Delta \eta|,|\Delta \phi|>0.5$ is divided by the rate of an L1 muon trigger requiring $p_{T}>10 \mathrm{GeV}$

\section{Validation}

\subsection{Hardware and Firmware Validation}

The execution of the L1Topo algorithms was tested in several ways. One such test was to compare the hardware output with software simulations, which were accurate to $\mathrm{O}(1 \%)$ as shown in Fig. 5. These results were further improved with bitwise simulations that account for the fact that the simulation runs on a floating point architecture, while the algorithms are executed on FPGAs capable of only integer arithmetic.

\section{Conclusions}

The demands placed on the L1Topo subsystem, such as low latency, highthroughput and computational accuracy, have all been met. The commissioning and validation of L1Topo has successfully demonstrated that despite the stringent bandwidth, latency, and hardware constraints, topological information can be extracted and utilized to improve the capabilities of the ATLAS trigger system. With 


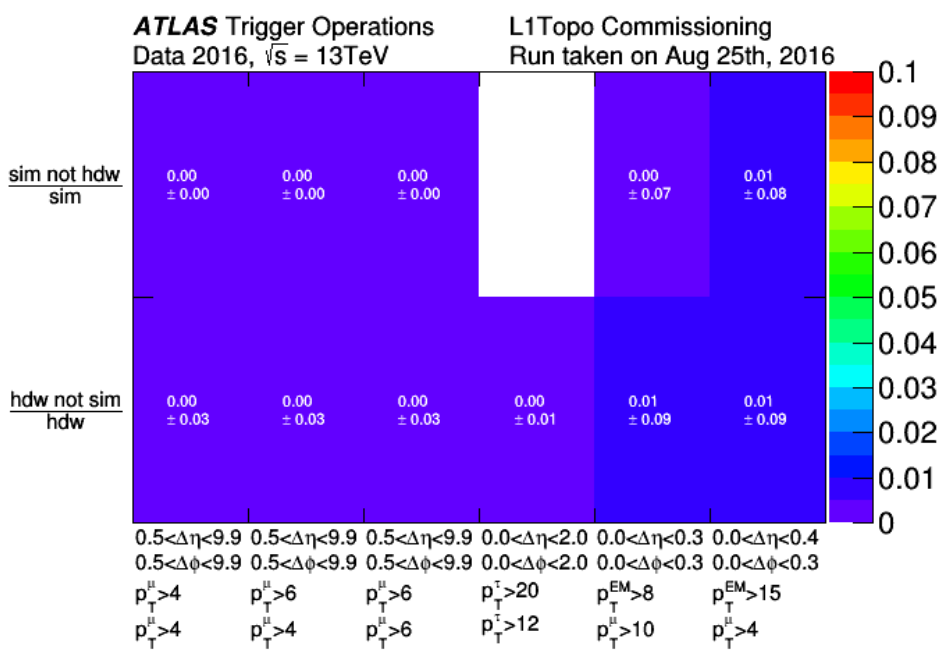

Fig. 5. Detailed comparison of event mismatches in simulation and hardware. $\mathrm{O}(1 \%)$ error are shown for false positives (top) and false negatives (bottom) for various L1Topo algorithms. $[5$

the introduction of the Level-1 Topological Trigger, the ATLAS trigger system is now capable of taking data at high luminosity with manageable rates while maintaining efficiency for several signatures relevant to important physics analyses.

\section{References}

1. ATLAS Collaboration, The ATLAS Experiment at the CERN Large Hadron Collider, (2008, JINST 3 S08003).

2. ATLAS Collaboration, Performance of the ATLAS Trigger System in 2010, (2011, arXiv:1110.1530).

3. ATLAS TDAQ Collaboration, The ATLAS Data Acquisition and High Level Trigger system, (2008, JINST 11 P06008).

4. E. Simioni, The Topological Processor for the future ATLAS Level-1 Trigger: from design to commissioning, (2014, arXiv:1406.4316).

5. ATLAS Collaboration, Trigger Operation Public Results, (2017) https://twiki.cern. ch/twiki/bin/view/AtlasPublic/TriggerOperationPublicResults

6. ATLAS Collaboration, ATLAS B-physics studies at increased LHC luminosity, potential for CP-violation measurement in the $B_{s}^{0} \rightarrow J / \psi \phi$ decay, (2013, ATL-PHYS-PUB2013-010)

7. F. Anulliet al., The Level-1 Trigger Muon Barrel System of the ATLAS experiment at CERN, (2009, JINST4P0401)

8. W.T. Fedorak and P.P. Plucinski, CMX Base Function FPGA Firmware Functionality, (2015)

9. S. Artz Physics performance with the new ATLAS Level-1 Topological trigger in Run 2, (2016, Proc. of the Fourth Annual Large Hadron Collider Physics, ATL-DAQ-PROC2016-012) 